\title{
A 1.9-GHz CMOS Power Amplifier Using Three-Port Asymmetric Transmission Line Transformer for a Polar Transmitter
}

\author{
Changkun Park, Student Member, IEEE, Younsuk Kim, Student Member, IEEE, Haksun Kim, Member, IEEE, and \\ Songcheol Hong, Member, IEEE
}

\begin{abstract}
A 1.9-GHz CMOS differential power amplifier for a polar transmitter is implemented with a $0.18-\mu \mathrm{m}$ RF CMOS process. All of the matching components, including the input and output transformers, are fully integrated. The concepts of injection locking and variable load are applied to increase the efficiency and dynamic range of the amplifier. An asymmetric three-port transmission line transformer is proposed to embody the variable load effectively. The power amplifier achieved a power-added efficiency of $40 \%$ at a maximum output power of $32 \mathrm{dBm}$. The dynamic range was $20 \mathrm{~dB}$ at supply voltages ranging from 0.5 to $3.3 \mathrm{~V}$. The improvement of the low power efficiency was $290 \%$ at an output power of $16 \mathrm{dBm}$.
\end{abstract}

Index Terms-Class-E, CMOS, dynamic range, global system for mobile communication (GSM), injection locking, polar transmitter, power amplifier, transmission line transformer, variable load.

\section{INTRODUCTION}

$\mathbf{P}$ OLAR transmitters are expected to be very popular owing to their many advantages over conventional Cartesian transmitters, especially in global system for mobile communications (GSM) and EDGE systems. Accordingly, their power amplifiers based on GaAs HBTs are being studied intensively. However, few studies have focused on CMOS power amplifiers for polar transmitters. Although CMOS power amplifiers are expected to be cheaper than GaAs HBT power amplifiers and easier to integrate with other circuits, they are not considered to be a useful RF power amplifier with a watt-level output power. Recently, the potential of a CMOS power amplifier was successfully demonstrated using a distributed active transformer [1]-[3]. The distributed active transformer is considered to have the potential to lend improvements to the performance of a CMOS power amplifier [4]. In this study, the concept of the distributed active transformer is used to design a CMOS power amplifier for polar transmitter applications.

Manuscript received July, 13, 2006; revised October, 19, 2006. This work was supported by the Korea Science and Engineering Foundation under the Engineering Research Center Program through the Intelligent Radio Engineering Center in Korea.

C. Park and S. Hong are with the Department of Electrical Engineering and Computer Science, Korea Advanced Institute of Science and Technology, Daejeon 305-701, Korea (e-mail: pck77@eeinfo.kaist.ac.kr).

Y. Kim was with the Department of Electrical Engineering and Computer Science, Korea Advanced Institute of Science and Technology, Daejeon 305701, Korea. He is now with the Samsung Electro-Mechanics Company Ltd., Suwon 443-803, Korea.

H. Kim is with the Department of Radio-Wave Engineering, Hanbat National University, Daejeon 305-719, Korea.

Digital Object Identifier 10.1109/TMTT.2006.889152

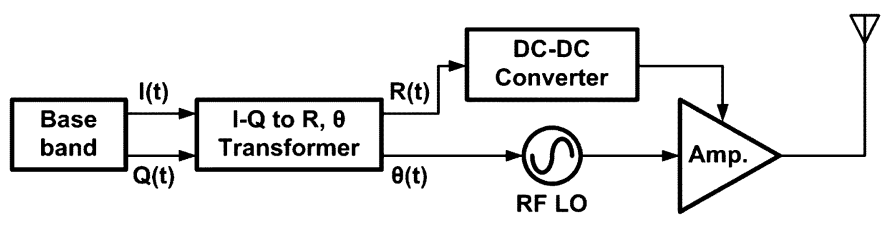

Fig. 1. Simplified block diagram of a polar transmitter.

Fig. 1 shows a block diagram of a polar transmitter. The polar transformer ( $I-Q$ to $R-\theta$ ) decomposes the input of a power amplifier into two types of input signals. The first of these is an RF phase signal, which is applied to the input of a power amplifier, and the second is an envelope signal, applied as a supply voltage $\left(V_{D D}\right)$. The supply voltage has to be applied through a dc-dc converter or a low drop output regulator circuit in order to supply sufficient power. Polar transmitters can use switching-mode power amplifiers, as the input signals do not contain envelope information. These include class-D, class-E, and class-F amplifiers, which are nonlinear, but very efficient. A CMOS power transistor is known to be viable for switching power amplifiers rather than linear amplifiers; thus, it common to study CMOS switching power amplifiers for polar transmitters [1]-[3], [5]. There are two important specifications of power amplifiers for polar transmitters. The first is the output dynamic range. It is crucial to obtain enough dynamic range with a given supply voltage range. The second specification is related to efficiency at a low output power. In general, a power efficiency close to the maximum output power is fairly high. However, the efficiency at a low output power is very low. Therefore, a stage-convertible power amplifier using a variable load method [6] is proposed as a means of increasing the dynamic range of the power amplifiers and improving the efficiency at a low output power. An asymmetric three-port transmission line transformer is also proposed to embody the variable load method efficiently.

\section{LOAD IMPEDANCE TRANSFORMATION}

\section{A. Power Efficiency of Class-E Power Amplifiers}

Polar transmitters can use switching-mode power amplifiers, as mentioned in Section I. The power efficiencies of polar transmitters are, therefore, expected to be higher than those of conventional transmitters; however, in a general switching mode, power amplifiers require a driving power greater than that for linear amplifiers. In a conventional class-E power amplifier, the 


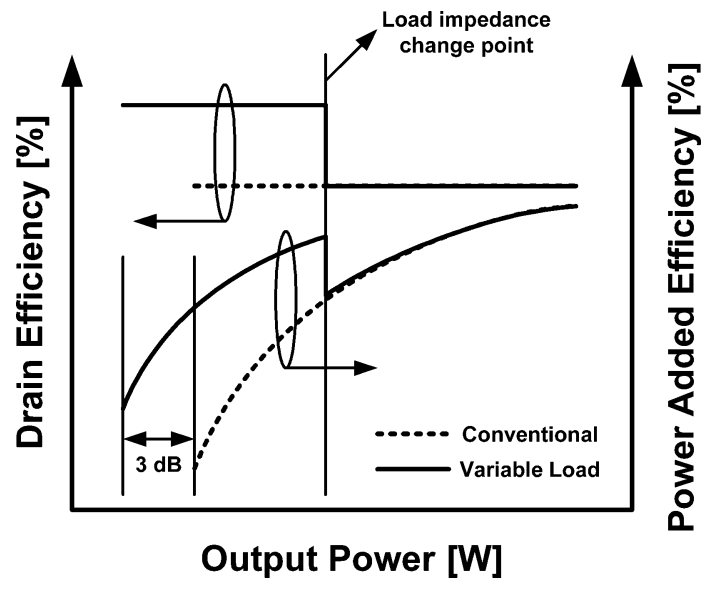

Fig. 2. Drain efficiency and PAE of a conventional and a variable load power amplifier.

power consumption of the driver stage degrades the overall efficiency severely regardless of the high drain efficiency of the power amplifier.

As the output power of a conventional class-E power amplifier is in proportion to $V_{D D}^{2}$ with a fixed input power, as shown by

$$
P_{\text {OUT }}(W) \propto \frac{V_{D D}^{2}}{R_{\text {load }}}
$$

the gain is decreased severely at a low $V_{D D}$ [7]. Although the drain efficiency maintains a high value, the power-added efficiency (PAE) of the power amplifier is degraded as the $V_{D D}$ decreases. The drain efficiency and PAE of a power amplifier are expressed as follows:

$$
\begin{aligned}
\text { Drain Efficiency } & \propto \frac{R_{\text {load }}}{R_{\text {on }}+R_{\text {load }}} \\
\text { PAE } & \propto \frac{R_{\text {load }}}{R_{\text {on }}+R_{\text {load }}} \cdot\left(1-\frac{P_{\mathrm{IN}}(W)}{P_{\text {OUT }}(W)}\right)
\end{aligned}
$$

where $P_{\mathrm{IN}}$ is the input power, $P_{\mathrm{OUT}}$ is the output power, $R_{\mathrm{on}}$ is the on resistance of the power transistors, and $R_{\text {load }}$ is the load impedance of the power amplifier. Fig. 2 shows the drain efficiency and PAE versus the $P_{\text {OUT }}$ of a conventional power amplifier, and a variable load power amplifier when the $V_{D D}$ varies with a fixed $P_{\mathrm{IN}}$. As the output power is in proportion to $V_{D D}^{2}$, as shown in (1), the drain efficiency and PAE versus $P_{\text {OUT }}$ can be determined, as shown in Fig. 2. The dotted lines denote the drain efficiency and PAE of a conventional power amplifier. If the low load impedance is increased two times in the low output power region, the drain efficiency and PAE are increased according to (2) and (3), respectively. The drain efficiency and PAE curves with variable loads appear as solid lines in Fig. 2.

Although a low load impedance is required to obtain high maximum output power, the load impedance must be increased as $V_{D D}$ is decreased in order to increase the efficiency. The variable load allows a high maximum output power at high power and a high efficiency at low power.

\section{B. Dynamic Range of Class-E Power Amplifiers}

The dynamic range of a power amplifier is the difference between the maximum output power and the minimum output

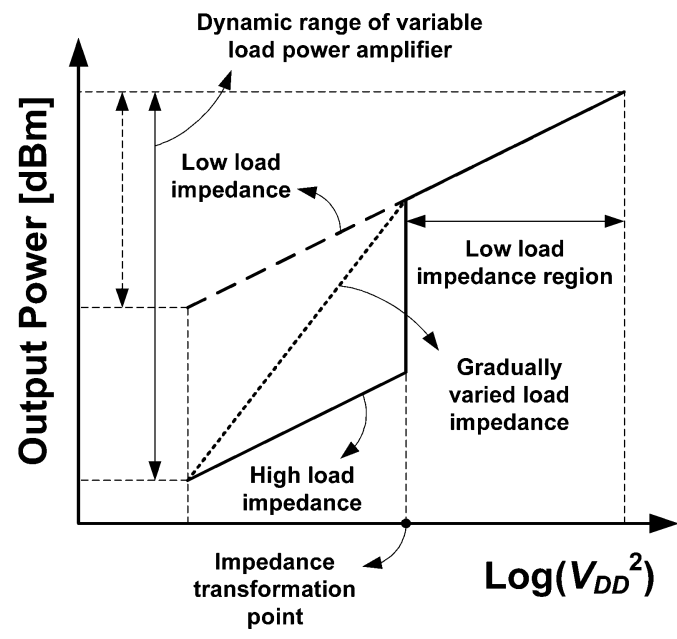

Fig. 3. Dynamic range of a power amplifier with a variable load.

power. As the load impedance of a conventional class-E power amplifier is fixed, the dynamic range of the conventional power amplifier for polar transmitters is determined by the variable range of $V_{D D}$. To satisfy the dynamic range of a GSM system, i.e., $20 \mathrm{~dB}$, the minimum supply voltage must be as low as $0.33 \mathrm{~V}$ with a maximum supply voltage of $3.3 \mathrm{~V}$. However, $0.33 \mathrm{~V}$ is too low to generate with a dc-dc converter. Additionally, such a low supply voltage degrades the overall efficiency of a dc-dc converter. Moreover, the phase distortion and the feed-through of the power amplifier are significant issues under a low supply voltage [8].

To solve these problems, a variable load method can be applied to the power amplifier for a polar transmitter. As the output power is in proportion to $V_{D D}^{2}$, the output power is decreased as $V_{D D}$ is decreased. If the load impedance is increased at a low $V_{D D}$ region, the dynamic range can be increased, as shown by

$$
\begin{aligned}
\text { Dynamic range } & =P_{\text {OUT max }}(\mathrm{dBm})-P_{\text {OUT min }}(\mathrm{dBm}) \\
& =10 \log \frac{\left(\frac{V_{D D_{\max }}^{2}}{R_{L}}\right)}{\left(\frac{V_{D D_{\min }}^{2}}{R_{H}}\right)} \\
& =20 \log \frac{V_{D D_{\max }}}{V_{D D_{\min }}}+10 \log \frac{R_{H}}{R_{L}} \\
& =20 \log \frac{3.3}{0.7}+10 \log \frac{15}{3} \\
& \cong 20.5 \mathrm{~dB} .
\end{aligned}
$$

In this equation, the parameter $V_{D D \max }$ is the maximum $V_{D D}, V_{D D \text { min }}$ is the minimum $V_{D D}, R_{L}$ is the low load impedance for the high output power, and $R_{H}$ is the high load impedance for the low output power. As shown in (4), the dynamic range can be controlled by not only the range of variable $V_{D D}$, but also through the ratio between $R_{L}$ and $R_{H}$. Assuming the values of $3.3 \mathrm{~V}$ for $V_{D D \max }, 0.7 \mathrm{~V}$ for $V_{D D \min }$, and $3 \Omega$ for $R_{L}$, the required load impedance ratio $R_{H} / R_{L}$ should be 5 for the dynamic range of a GSM system.

Fig. 3 explains (4). At a certain point of $V_{D D}$, the load impedance $R_{\text {load }}$ changes from low to high. As $P_{\text {OUT }}$ versus $V_{D D}^{2}$ decreases as $R_{\text {load }}$ increases, as shown in (1), the dynamic 


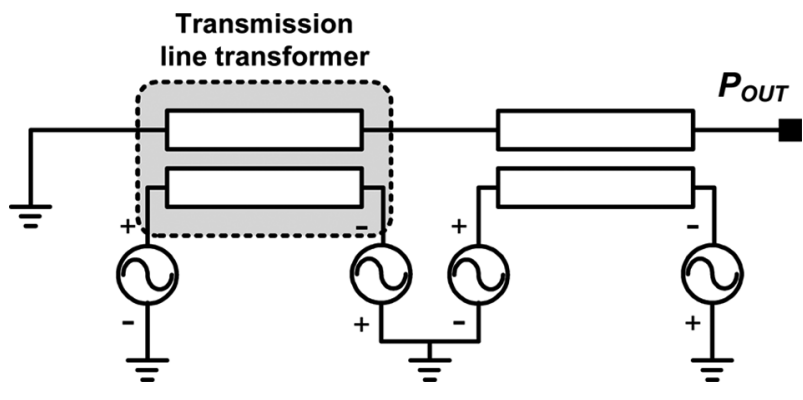

Fig. 4. Simplified schematic of the output transformer (after [1] and [2]).

range increases for a given range of $V_{D D}$. In Fig. 3, the dashed line shows $P_{\text {OUT }}$ of a conventional power amplifier. The solid line in this figure shows $P_{\text {OUT }}$ of a power amplifier with an abruptly varied load, and the dotted line shows $P_{\text {OUT }}$ of a power amplifier with a gradually varied load. The dotted arrow shows the dynamic range of a conventional power amplifier and the solid arrow shows the dynamic range of a power amplifier with a variable load. It is important to note that the dynamic range can be extended using the variable load method.

\section{Proposed Three-Port Asymmetric TRANSMISSION LINE TRANSFORMER}

\section{A. Impedance Transformation}

In contrast to GaAs technology, there is no via process in CMOS technology. Thus, a bond wire is needed to make an ac ground in RF CMOS circuits. By applying a differential structure, it is possible to obtain virtual grounds, thereby preventing the gain reductions that are induced by the bond wires.

However, in general, the antenna and filter connected to the output of the power amplifier are single-ended components. Thus, a transformer is needed to connect the differential power amplifier to the single-ended components. In this study, a high- $Q$ transmission line transformer is used as the output transformer. The transformer significantly influences the output power and efficiency. The output matching is completed with an additional metal-insulator-metal (MIM) capacitor. Two differential pairs of a power stage are used, as shown in Fig. 4.

If the $k$-factor of the transformer used in Fig. 4 is 1 , as is ideal, and there is no parasitic inductance in the transmission line transformer, the load impedance of $50 \Omega$ is transformed into $12.5 \Omega$ with an impedance transforming ratio of 1 to 4 [1], [2]. However, the inductance of the transmission line transformer plays an important role in the output matching network. To verify the role of the transmission line transformer in the output matching network, Fig. 5 shows an equivalent circuit for the output matching network, which is composed of the transmission line transformer and additional MIM capacitors $C$ and $C_{\text {shunt }}$. To simplify the analysis, the turn ratio of the transmission line transformer, which is used in the analysis, is 1 , as shown in Fig. 5(a).

Fig. 5(b) shows the equivalent circuit of the output matching network shown in Fig. 5(a). $L_{1}$ and $L_{2}$ are the self-inductances. The parasitic inductances of the transmission line transformer,

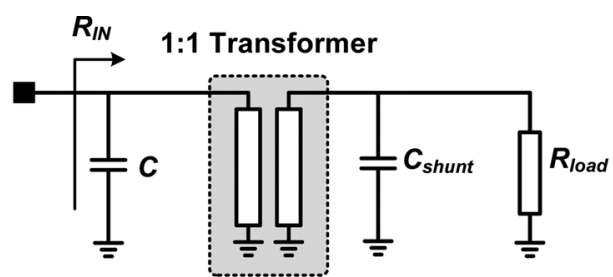

(a)

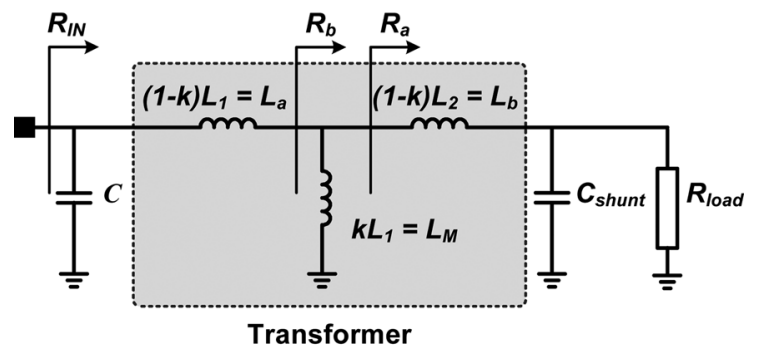

(b)

Fig. 5. (a) Output matching network. (b) Equivalent circuit for the output matching network.

e.g., $L_{a}, L_{b}$, and $L_{M}$ in Fig. 5(b), can be used as output matching components. The following equations demonstrate the process of impedance transformation using the transmission line transformer and the additional capacitors, $C$ and $C_{\text {shunt }}$. $R_{a}$ of Fig. 5 can be calculated as follows:

$$
\begin{aligned}
R_{a}=\frac{R_{\text {load }}}{1+} & \left(\omega R_{\text {load }} C_{\text {shunt }}\right)^{2} \\
& +j \omega \cdot \frac{L_{b}+\omega^{2} R_{\text {load }}^{2} L_{b} C_{\text {shunt }}^{2}-R_{\text {load }}^{2} C_{\text {shunt }}}{1+\left(\omega R_{\text {load }} C_{\text {shunt }}\right)^{2}} .
\end{aligned}
$$

The imaginary part of (5) is equal to zero at resonance with $L_{b}$ and $C_{\text {shunt }}$, as described in

$$
L_{b}+\omega^{2} R_{\text {load }}^{2} L_{b} C_{\text {shunt }}^{2}-R_{\text {load }}^{2} C_{\text {shunt }}=0 .
$$

Here, $L_{b}$ is expressed by

$$
L_{b}=\frac{R_{\text {load }}^{2} C_{\text {shunt }}}{1+\left(\omega R_{\text {load }} C_{\text {shunt }}\right)^{2}} .
$$

$R_{b}$ and $R_{\mathrm{IN}}$ are calculated by

$$
\begin{aligned}
R_{b} & =\frac{j \omega R_{a} L_{M}}{R_{a}+j \omega L_{M}}=\frac{\omega^{2} R_{a} L_{M}^{2}}{R_{a}^{2}+\omega^{2} L_{M}^{2}}+j \omega \cdot \frac{R_{a}^{2} L_{M}}{R_{a}^{2}+\omega^{2} L_{M}^{2}} \\
R_{\mathrm{IN}} & =\frac{\omega^{2} R_{a} L_{M}^{2}+j A}{R_{a}^{2}\left\{1-\omega^{2} C\left(L_{a}+L_{M}\right)\right\}^{2}+\omega^{2} L_{M}^{2}\left(1-\omega^{2} L_{a} C\right)^{2}} .
\end{aligned}
$$

Afterwards, the imaginary part of (9) can be equal to zero using the value $C$ from Fig. 5, as described in

$$
\begin{aligned}
& A=\omega^{3} L_{a} L_{M}^{2}+\omega R_{a}^{2}\left(L_{a}+L_{M}\right) \\
& -C\left\{\omega^{5} L_{a}^{2} L_{M}^{2}+\omega^{3}\left(L_{a}+L_{M}\right)^{2} R_{a}^{2}\right\}=0 .
\end{aligned}
$$

Following this, the value $C$ can be expressed as

$$
\therefore C=\frac{\omega^{3} L_{a} L_{M}^{2}+R_{a}^{2}\left(L_{a}+L_{M}\right)}{\omega^{4} L_{a}^{2} L_{M}^{2}+\omega^{2}\left(L_{a}+L_{M}\right)^{2} R_{a}^{2}} \text {. }
$$




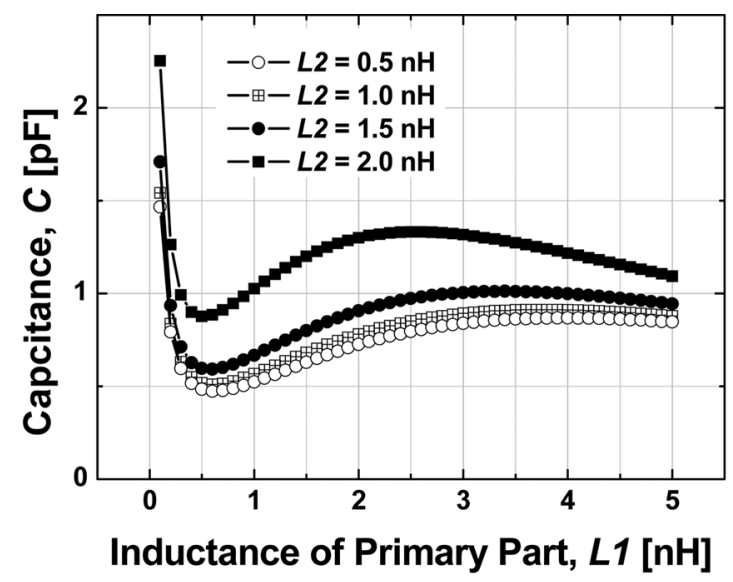

(a)

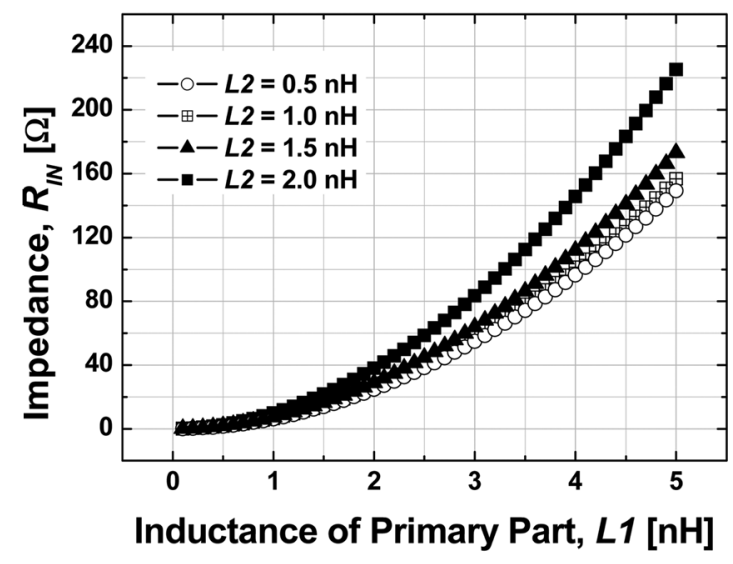

(b)

Fig. 6. Simulation results of: (a) the capacitance $C$ and (b) the transformed load impedance $R_{\mathrm{IN}}$.

$R_{\mathrm{IN}}$ can, therefore, be expressed by

$$
R_{\mathrm{IN}}=\frac{\omega^{2} R_{a} L_{M}^{2}}{R_{a}^{2}\left\{1-\omega^{2} C\left(L_{a}+L_{M}\right)\right\}^{2}+\omega^{2} L_{M}^{2}\left(1-\omega^{2} L_{a} C\right)^{2}} .
$$

Assuming the $k$-factor is 0.5 and $R_{\text {load }}$ is $25 \Omega$, the transformed load impedance $R_{\mathrm{TN}}$ versus the varied inductance of the primary part was simulated using (12). The inductance of the secondary part $L_{2}$ is assumed to be $0.5,1.0,1.5$, and $2.0 \mathrm{nH}$ in the simulation. Fig. 6 shows simulated $C$ and $R_{\mathrm{IN}}$. As shown in Fig. 6(b), $R_{\mathrm{IN}}$ increases as $L_{1}$ increases. Thus, although the impedance transformation ratio of the ideal transformer is 1 , the impedance transformation ratio of the transmission line transformer can be controlled by the parasitic component of the transformer. From the results shown in Fig. 6, the inductance of the primary part must be small in order to obtain the low load impedance for a high maximum output power. As shown in Section II, the inductance of the primary part must be large in order to obtain a high load impedance for a high dynamic range and a high efficiency at the low output power region.

It is interesting to note that, for a high load impedance, the $k$-factor of the transmission line transformer does not increase as the inductance $L_{1}$ increases. If the $k$-factor increases with

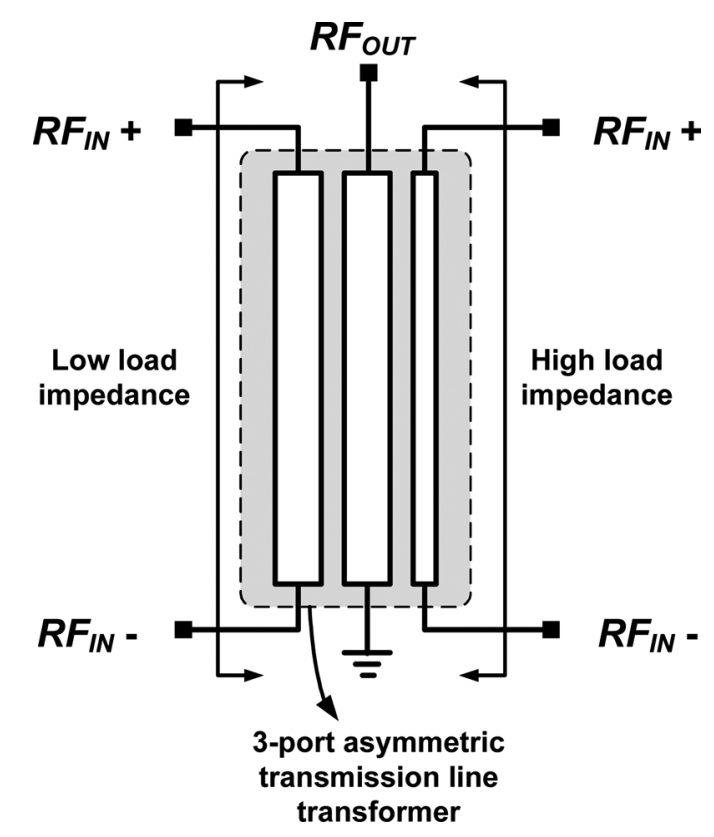

Fig. 7. Simplified schematic of the proposed three-port asymmetric transmission line transformer.

an increase of $L_{1}, R_{\mathrm{IN}}$ cannot be increased effectively as $L_{1}$ increases.

\section{B. Implementation of a Three-Port Asymmetric Transmission Line Transformer}

A three-port transmission line transformer is proposed in order to obtain a low and high load impedance with a transformer, as simplified in Fig. 7. The primary part of the transformer consists of two transmission lines, which have different inductances. The inductances can be controlled by the length and width of the slab waveguides. One of these is designed for a low load impedance to obtain a high maximum output power. The other is designed for a high load impedance in order to obtain a high dynamic range and high efficiency at a low output power. The proposed three-port asymmetric transmission line transformer, therefore, provides two load impedances.

The $k$-factor of the primary high-impedance transmission line to the secondary line should not exceed that of the low-impedance line in order to obtain the two load impedances effectively. If the $k$-factor of the high load impedance part exceeds that of the low load impedance part, the value of $L_{a}$ of the high load impedance part cannot be higher than that of $L_{a}$ of the low load impedance part. In this study, a three-port transmission line transformer is embodied, as shown in Fig. 8. The primary part for the low-power mode has spiral turns to increase the parasitic inductance of the transformer. The proposed three-port transmission line transformer is applied to the proposed power amplifier for a polar transmitter application in order to achieve a high maximum output power, high dynamic range, and high efficiency at a low output power region. The simulated loss of the transformer itself for a high power mode is approximately $1.7 \mathrm{~dB}$. 


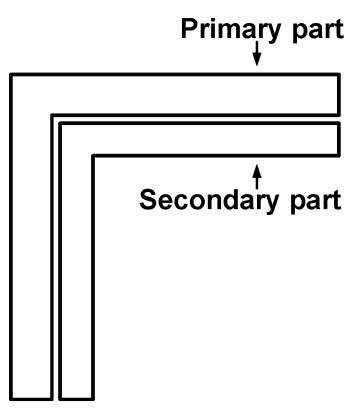

(a)

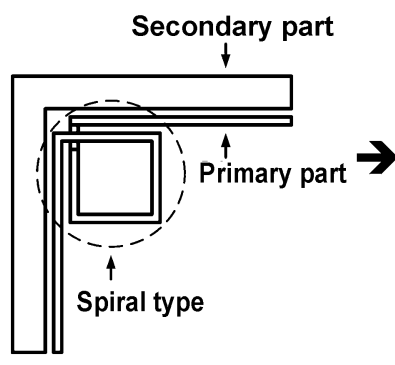

(b)

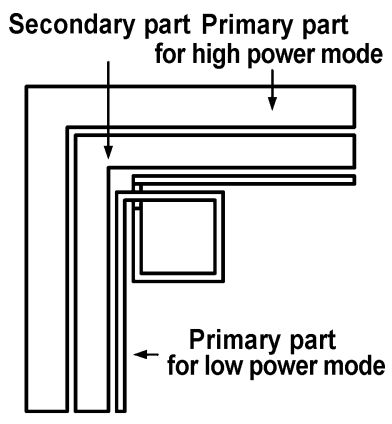

(c)

Fig. 8. Three-port asymmetric transmission line transformer. (a) Transformer for a high-power mode. (b) Transformer for a low-power mode. (c) Three-port transmission line transformer.

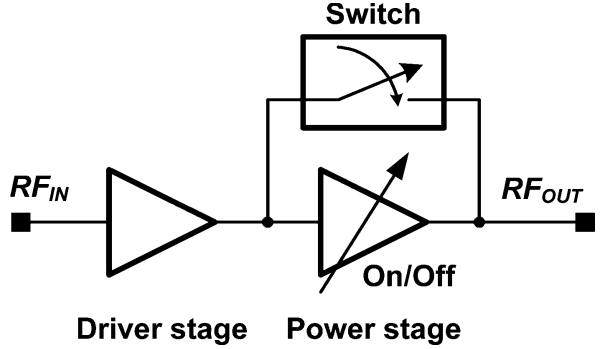

Fig. 9. Power amplifier with the bypass switch.

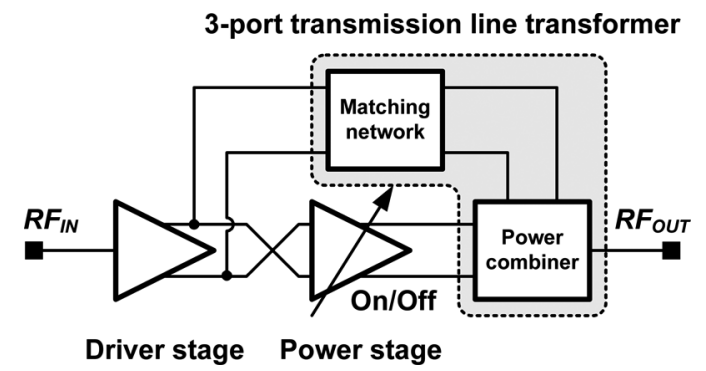

Fig. 10. Proposed stage-convertible power amplifier architecture.

\section{DESIGN OF THE 1.9-GHz CMOS POWER AMPLIFIER}

\section{A. Proposed Stage-Convertible Power Amplifier}

The proposed stage-convertible power amplifier is a modified structure of a power amplifier with a bypass switch, as shown in Fig. 9 [9]. The switch is located parallel to the power stage, as shown in Fig. 9. The power stage is turned on and the switch is turned off to achieve the maximum gain and output power. For the low output power, the power stage is turned off and the switch is turned on to bypass the power stage. The output of driver stage then becomes the output of the power amplifier.

In the proposed power amplifier, a low-power matching network is located parallel to the power stage. The output of the power stage and the output of the matching network are combined in a power combiner, as shown in Fig. 10. For a high-power mode, the power stage is turned on and the driver stage drives the power stage. In addition, a certain amount of driver power is coupled to the output directly through the three-port transmission line transformer. The load impedance for the power stage is low to achieve a high maximum output
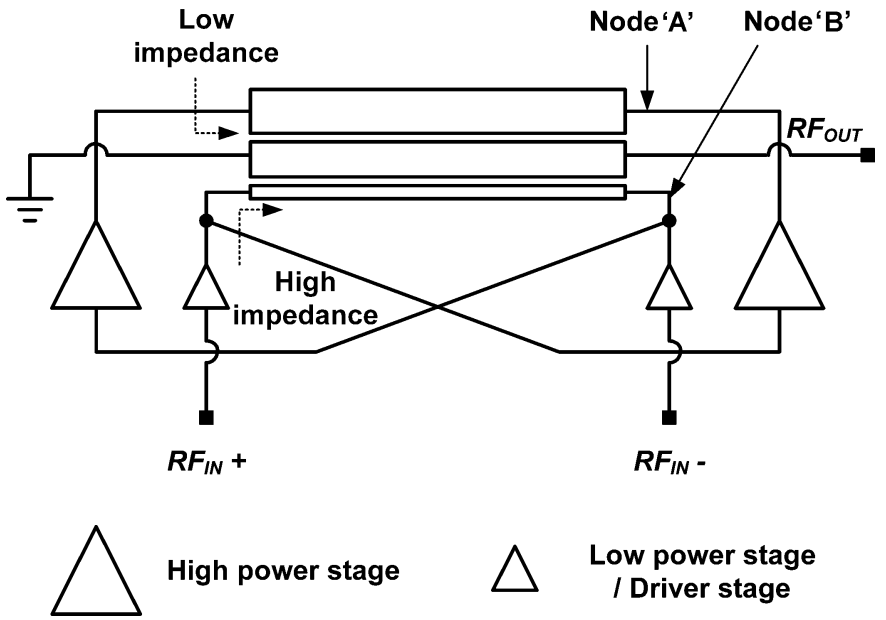

High power stage

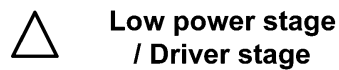

Fig. 11. Schematic of the stage-convertible power amplifier.

power. For a low-power mode, the power stage is turned off and only power from the driver is transmitted into the output port through the low-power matching network. The load impedance of the driver stage must be high in order to obtain a high dynamic range in addition to a high efficiency in the low output power region. In the proposed stage-convertible power amplifier, high- and low-power modes of the power amplifier are selected by turning on, and off, respectively, the power stage. The power combiner and low-power matching network are implemented with the three-port transmission line transformer, as shown in Fig. 11. A schematic of the proposed power amplifier is shown in Fig. 11, which has a differential structure. The driver and power stages were designed as class-E amplifiers. The output of the power stage is connected to the one primary part of the transformer that has a low load impedance. The output of the driver stage is connected not only to the input of the power stage, but also to the other primary part of the transformer with a high load impedance.

For the high-power mode, all of the stages in the power amplifier are turned on to generate a high output power. For the low-power mode, the power stage is turned off and the output power is generated only by the driver stage. The output power of the driver stage is transmitted into the output while in the low-power mode. In Fig. 11, a pair of differential power stages and a pair of differential driver stages are cross-coupled to create 


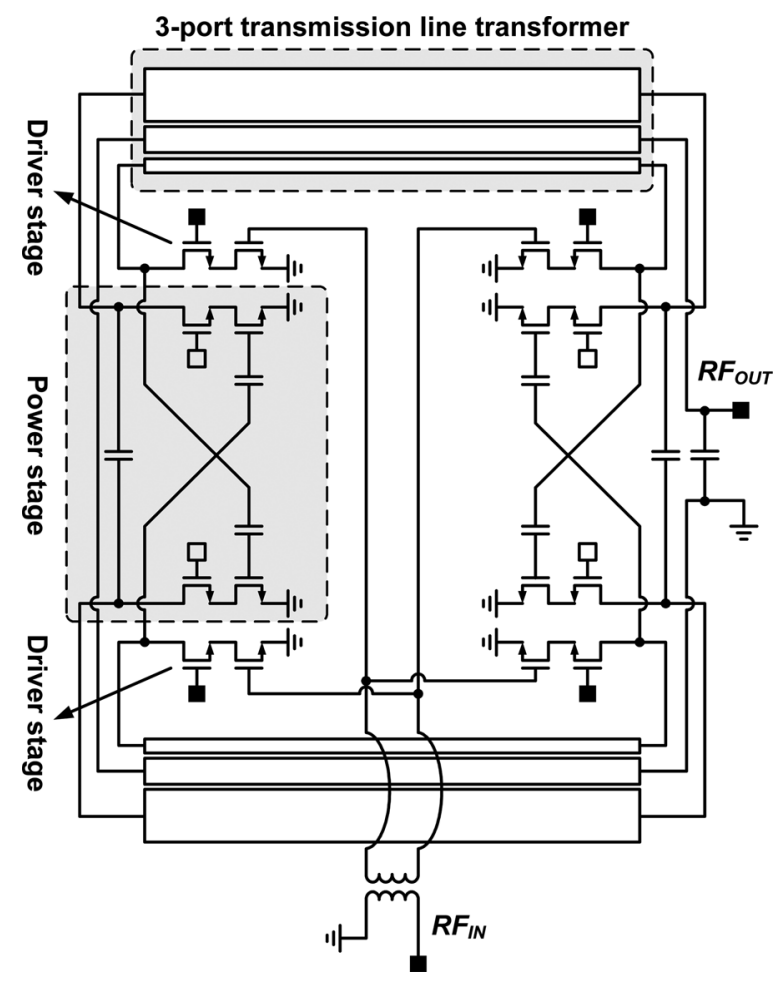

Fig. 12. Schematic of the designed power amplifier.

node " $A$ " and node " $B$ " in the phase. In the proposed power amplifier, an additional power stage is not needed for the lowpower mode. The driver stage works as the output stage in the low-power mode. The chip size is, therefore, reduced.

A schematic of the designed power amplifier is shown in Fig. 12. The concept of a distributed active transformer is used to realize the voltage-combining technique. Two differential pairs of the power stage and the driver stage are used. The output matching network of the power stage is implemented with the three-port transformers, the drain-source capacitance of the power stage, and an additional MIM capacitor. The output matching network of the driver stage is composed of the transformers, the drain-source capacitance of the driver stage, the gate-source capacitance of the power stage, and an additional MIM capacitor. The input transformer consists of a spiral-type transformer.

\section{B. Auto-Switching Technique}

An auto-switching technique is used to switch to the highand low-power modes automatically with respect to the $V_{D D}$ of the power amplifier, which is an amplitude signal of a polar transmitter. Therefore, an additional external control signal to change the mode of the power amplifier is not needed.

As mentioned in Section II, the output power of a polar transmitter is controlled via the variable $V_{D D}$ of the power amplifier. The output power is, therefore, increased as $V_{D D}$ is increased. Here, both the driver stage and power stage are turned on when the supply voltage becomes high. However, the driver stage is turned on and the power stage is turned off automatically when the supply voltage is low. A cascode structure is used in each stage to realize the auto-switching technique. A conventional

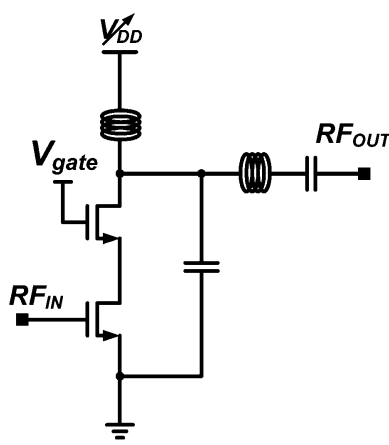

(a)

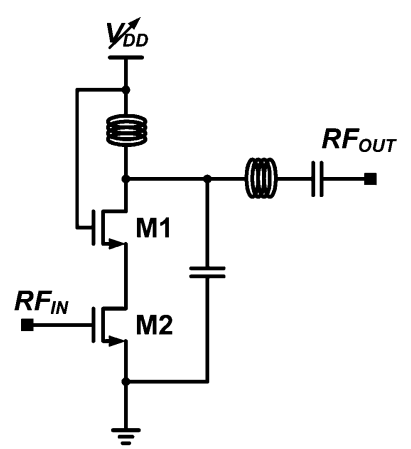

(b)
Fig. 13. Schematics of the power stages for: (a) conventional cascode structure and (b) self-biased cascode structure.

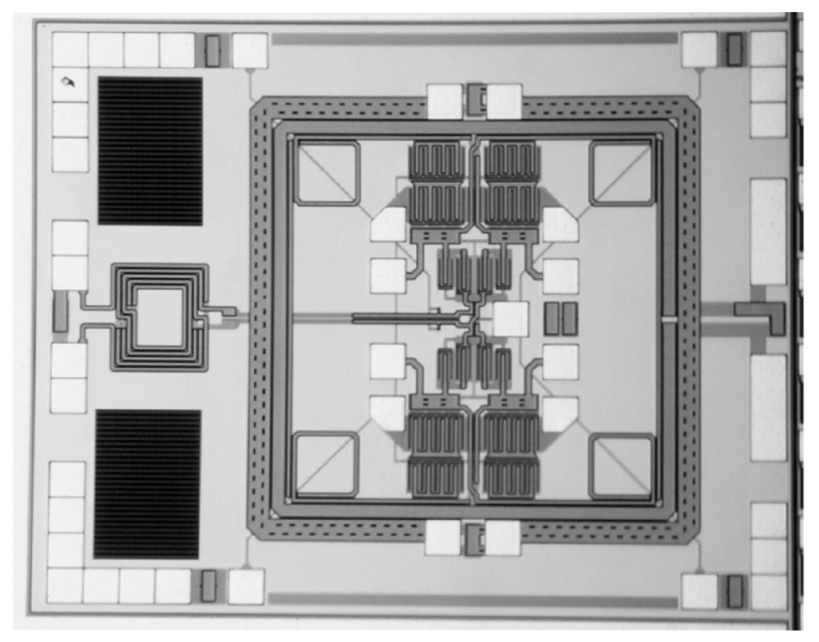

Fig. 14. Chip photograph of the implemented power amplifier.

cascode structure is shown in Fig. 13(a), and a self-biased cascode structure is shown in Fig. 13(b) [10]. If the supply voltage of the self-biased cascode structure is decreased, the on resistance of $M 1$ is increased and the current through the power transistor of the self-biased cascode structure becomes smaller than that of the conventional cascode structure.

In this study, the conventional cascode structure is used in the driver stage and the self-biased cascode structure is used in the power stage. The power stage is programmed to be turned on or turned off automatically according to the variable supply voltage. The contribution of the power stage to the output power is decreased and the contribution of the driver stage to the output power is increased as $V_{D D}$ is decreased. Thus, the high-power mode becomes dominant in the higher $V_{D D}$ region and the lowpower mode becomes dominant in the lower $V_{D D}$ region.

\section{Measurement Results}

Fig. 14 shows the implemented power amplifier using $0.18-\mu \mathrm{m}$ RF CMOS technology. The size of the chip is $1.2 \mathrm{~mm}$ $\times 1.8 \mathrm{~mm}$ including pads. All of the matching components, including the input and output transformers, are fully integrated. The area of the transformer is $1.0 \mathrm{~mm} \times 1.0 \mathrm{~mm}$. The metal for the transformer is $2.34-\mu \mathrm{m}$-thick aluminum. 


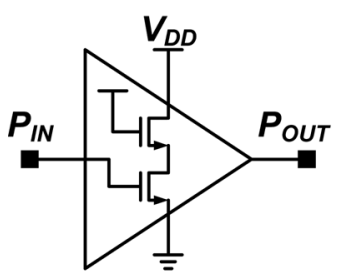

Driver Stage

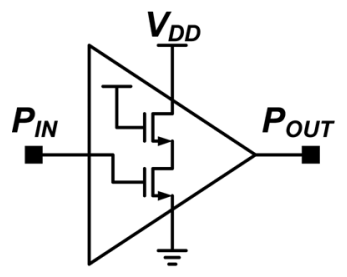

Driver Stage

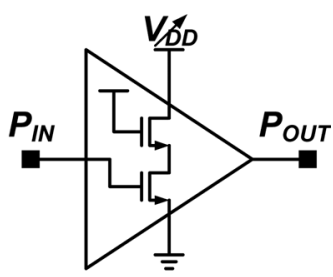

Power Stage

(a)

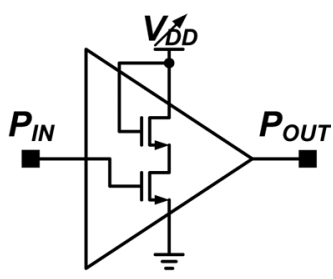

Power Stage (b)

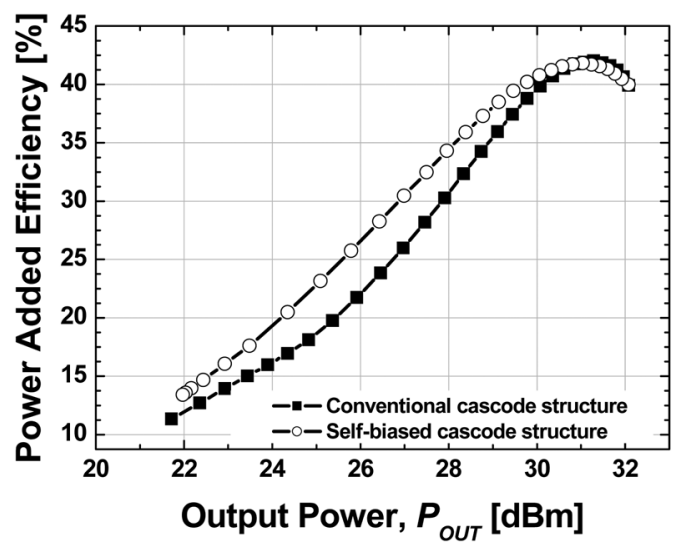

(c)

Fig. 15. Circuit configurations of: (a) conventional cascode structure, (b) selfbiased cascode structure, and (c) measurement results.

The losses of the bond-wire, input transformer, and printed circuit board interconnections are included in the amplifier's measured performance. Fig. 15 shows the measured PAE versus $P_{\text {OUT }}$, while $V_{D D}$ varies from 0.5 to $3.3 \mathrm{~V}$. In Fig. 15(a), conventional cascode structures are used in the power and driver stages, and $V_{D D}$ of the power stage varies while $V_{D D}$ of the driver stage remains fixed. In Fig. 15(a), the gate bias in the cascode transistor of the power stage is fixed at $3.3 \mathrm{~V}$. If the gate bias is lower than $3.3 \mathrm{~V}$, the maximum output power and PAE are degraded. In Fig. 15(b), a self-biased cascode structure is used in the power stage. The measured PAE of Fig. 15(b) is higher than that of Fig. 15(a). For the circuit configuration of Fig. 15(b), the contribution of the power stage decreases as $V_{D D}$ decreases. The efficiency of the structures in Fig. 15(b) in the low-power region is, therefore, higher than those in Fig. 15(a). The measured dynamic range is not sufficient for a GSM specification, and the efficiency improvement in the low output power region is limited.

To extend the dynamic range and to increase the efficiency further, $V_{D D}$ of the driver stage also varies with $V_{D D}$ of the power stage, as shown in Fig. 16. A measurement of the

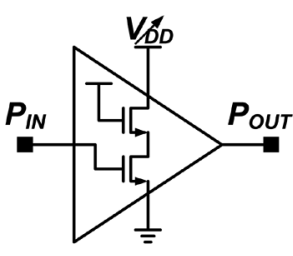

Driver Stage

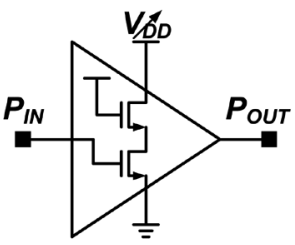

Driver Stage

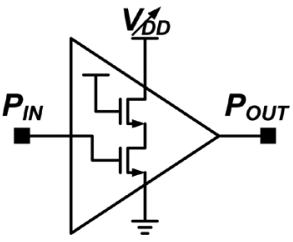

Driver Stage (a)

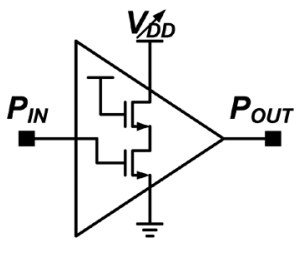

Power Stage

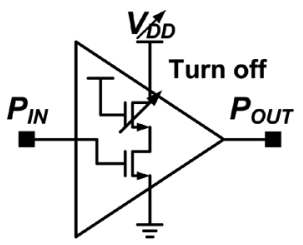

Power Stage (b)

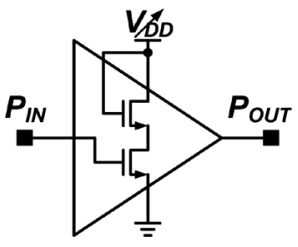

Power Stage

(c)

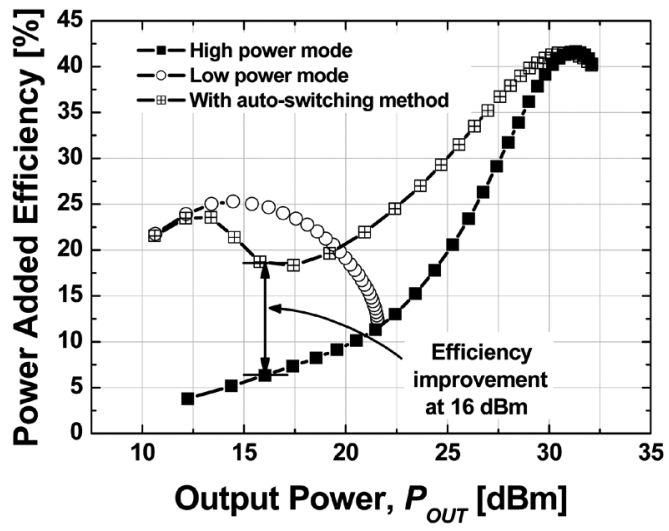

(d)

Fig. 16. Circuit configurations of: (a) high-power mode, (b) low-power mode, (c) auto-switching technique, and (d) measurement results.

high-power mode shows that the maximum output power is $32 \mathrm{dBm}$ with a PAE of $40 \%$. Theoretically, the dynamic range of a conventional class-E amplifier is approximately $16.4 \mathrm{~dB}$, while $V_{D D}$ varies from 0.5 to $3.3 \mathrm{~V}$. However, the dynamic range of the high-power mode is extended to nearly $20 \mathrm{~dB}$, as the input power of the power stage also decreases when $V_{D D}$ of the driver stage decreases. For the low-power mode, the power stage is turned off by an external signal. Due to the high load impedance of the low-power mode, the efficiency in a low output power region is increased. With the circuit configuration shown in Fig. 16(c), the auto-switching technique is realized by the self-biased cascode structure in the power stage. In the low output power region, the input power of the power stage is decreased. The contribution of the power stage is, therefore, decreased and the contribution of the driver stage that has a 


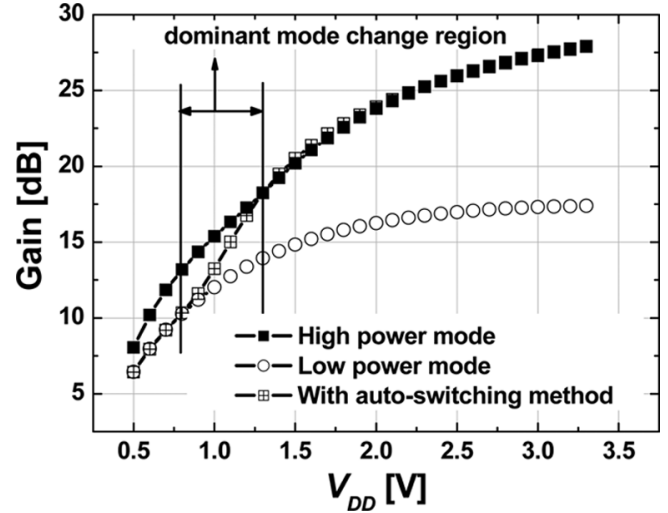

Fig. 17. Measured gains of different modes.

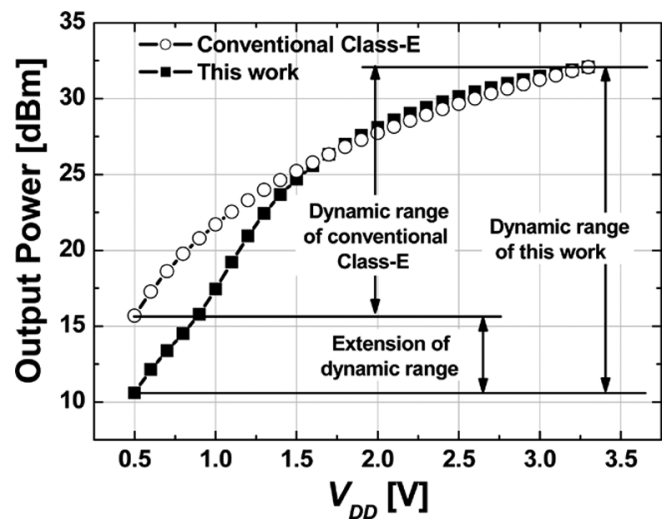

Fig. 18. Extension of dynamic range.

higher load impedance is increased. Thus, the power and driver stages are always turned on. As shown in Fig. 17, the mode of the power amplifier is changed automatically and smoothly with $V_{D D}$. This is due to the self-biased cascode structure of the power stage.

Fig. 17 shows the measured gains of different modes. For a given $V_{D D}$, the gain of a high-power mode always appears higher than that of a low-power mode due to the fixed input power of the class-E amplifier. As indicated in the graph, the mode of the power amplifier with the auto-switching method is changed automatically and smoothly in the mode-change region.

Fig. 18 shows an extension of the dynamic range. The white symbols show the theoretically calculated output power of a conventional class-E amplifier. The dynamic range of a conventional class-E amplifier is approximately $16.4 \mathrm{~dB}$, while $V_{D D}$ varies from 0.5 to $3.3 \mathrm{~V}$. The black symbols show the output power of the circuit configuration shown in Fig. 16(c). The measured extension of dynamic range is nearly $4 \mathrm{~dB}$.

Fig. 19 shows the frequency response under the maximum output power conditions. The single-ended output power is higher than $31 \mathrm{dBm}$ over a frequency range of $1.70-1.92 \mathrm{GHz}$. Fig. 20 shows the measured degradation of the output power with time under the maximum output power conditions.

The nonlinearities of a polar transmitter are mainly due to signal delay mismatches between amplitude path and phase

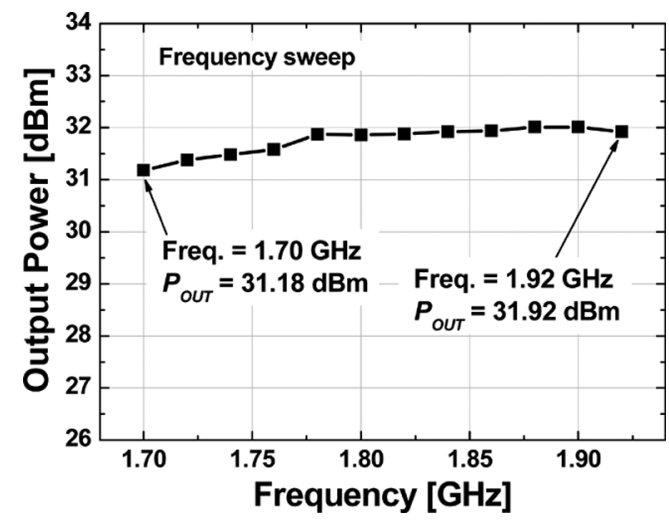

Fig. 19. Measured frequency response.

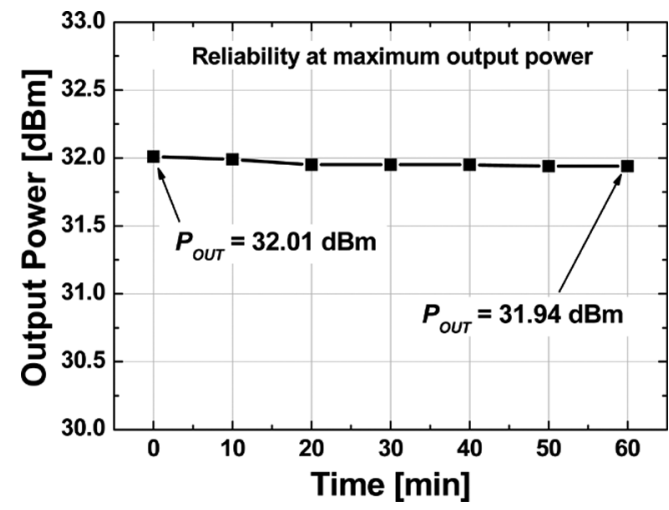

Fig. 20. Measured degradation of the output power with time.

path. There are also due to the AM-PM distortion of the amplifier. However, the nonlinearities can be solved using a digital predistorter in the polar transmitter.

\section{CONCLUSIONS}

A $1.9-\mathrm{GHz}$ power amplifier for a polar transmitter application has been implemented using $0.18-\mu \mathrm{m}$ RF CMOS technology. Stage-convertible power-amplifier architecture, an auto-switching technique, and a three-port asymmetric transmission line transformer have been proposed in order to improve efficiency and dynamic range. The improvement of the dynamic range is approximately $4 \mathrm{~dB}$, and the total dynamic range is nearly $20 \mathrm{~dB}$. The low-power efficiency improvement of the power amplifier is $290 \%$ at an output power of $16 \mathrm{dBm}$.

\section{REFERENCES}

[1] I. Aoki, S. D. Kee, D. B. Rutledge, and A. Hajimiri, "Distributed active transformer-A new power-combining and impedance-transformation technique," IEEE Trans. Microw. Theory Tech., vol. 50, no. 1, pp. 316-331, Jan. 2002.

[2] _ "Fully integrated CMOS power amplifier design using the distributed active-transformer architecture," IEEE J. Solid-State Circuits, vol. 37, no. 3, pp. 371-383, Mar. 2002.

[3] Y. Kim, C. Park, H. Kim, and S. Hong, "CMOS RF power amplifier with reconfigurable transformer," Electron. Lett., vol. 42, no. 7, pp. 405-407, Mar. 2006.

[4] S. Kim, K. Lee, J. Lee, B. Kim, S. D. Kee, I. Aoki, and D. B. Rutledge, "An optimized design of distributed active transformer," IEEE Trans. Microw. Theory Tech., vol. 53, no. 1, pp. 380-388, Jan. 2005. 
[5] A. Mazzanti, L. Larcher, R. Brama, and F. Svelto, “A $1.4 \mathrm{GHz}-2 \mathrm{GHz}$ wideband CMOS class-E power amplifier delivering $23 \mathrm{dBm}$ peak with $67 \%$ PAE," in IEEE Radio Freq. Integr. Circuits Symp. Dig., Jun. 2005, pp. $425-428$.

[6] A. Shirvani, "A CMOS RF power amplifier with parallel amplification for efficient power control," IEEE J. Solid-State Circuits, vol. 37, no. 6, pp. 684-693, Jan. 2002.

[7] N. O. Sokal and A. D. Sokal, "Class-E: A new class of high-efficiency tuned single-ended switching power amplifiers," IEEE J. Solid-State Circuits, vol. SSC-10, no. 6, pp. 168-176, Jun. 1975.

[8] P. Reynaert and M. S. J. Steyaert, "A 1.75-GHz polar modulated CMOS RF power amplifier for GSM-EDGE," IEEE J. Solid-State Circuits, vol. 40, no. 12, pp. 2598-2608, Dec. 2005.

[9] J. Staudinger, "Stage bypassing in multi-stage PAs," presented at the IEEE MTT-S Int. Microw. Symp. Workshop, Jun. 2000.

[10] T. Sowlati and D. M. W. Leenaerts, "A 2.4-GHz 0.18- $\mu \mathrm{m}$ CMOS selfbiased cascode power amplifier," IEEE J. Solid-State Circuits, vol. 38, no. 8, pp. 1318-1324, Aug. 2003.

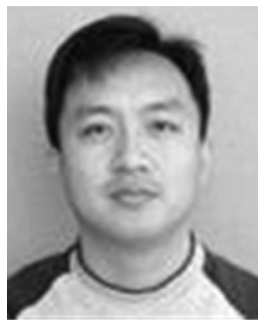

Younsuk Kim (S'03) was born in Seoul, Korea, in 1969. He received the B.A., M.A., and Ph.D. degrees in electronic engineering from the Korea Advanced Institute of Science and Technology (KAIST), Daejeon, Korea, in 1991, 1993, and 2006, respectively. His doctoral dissertation concerned CMOS RF power amplifiers with reconfigurable transformers.

From 1995 to 2002, he developed voltage-controlled oscillator (VCO) modules for mobile phone in Samsung electromechanics. From 2002 to 2006, he was a graduate student involved with projects concerning MMIC circuits and RF power amplifiers. He is currently with the Samsung Electro-Mechanics Company Ltd., Suwon, Korea, where he is involved with the development of CMOS power amplifiers and polar transmitters.

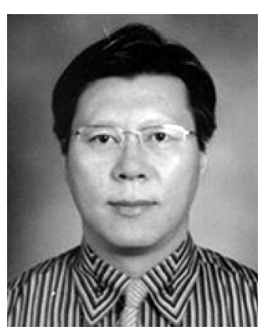

Haksun Kim (M'93) received the B.S., M.S., and $\mathrm{Ph} . \mathrm{D}$. degree in electronics from Hankuk Aviation University, Goyang City, Korea, in 1986, 1990, and 1993, respectively.

He has been with the Samsung Advanced Institute of Technology, the Ministry of Information and Communication, and Turbo Telecom. Since 1989, he has been a Professor with the Department of Radio-Wave Engineering, Hanbat National University, Daejeon, Korea. Since 2004, he has been Vice President of the Samsung Electro-Mechanics Company Ltd., Suwon, Korea. His research interests are circuits and systems, RF integrated-circuit design, which includes low/high date-rate wireless connectivity, wireless local area networks (WLANs), Wimax, millimeter waves, etc.

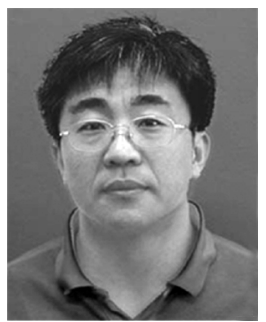

Songcheol Hong (S'87-M' 88 ) received the B.S. and M.S. degrees in electronics from Seoul National University, Seoul, Korea, in 1982 and 1984, respectively, and the $\mathrm{Ph} . \mathrm{D}$. degree in electrical engineering from The University of Michigan at Ann Arbor, in 1989.

In May 1989, he joined the faulty of the Department of Electrical Engineering and Computer Science, Korea Advanced Institute of Science and Technology (KAIST), Daejeon, Korea. In 1997, he held short visiting professorships with Stanford University and Samsung Microwave Semiconductor. His research interests are microwave integrated circuits and systems including power amplifiers for mobile communications, miniaturized radar, millimeter-wave frequency synthesizers, as well as novel semiconductor devices. 